\title{
Interação entre genótipos e ambientes em diferentes tipos de híbridos de milho
}

\author{
Emiliano Fernandes Nassau Costa(1), João Cândido de Souza(1), José Luis Lima(1) e Gustavo Andrade Cardoso(1) \\ (1)Universidade Federal de Lavras, Departamento de Biologia, Campus Universitário, Caixa Postal 37, CEP $37200-000$ Lavras, MG. \\ E-mail: emilianonassau@hotmail.com, cansouza@dbi.ufla.br, joseluis_li@yahoo.com.br, gustavoancardoso@hotmail.com
}

Resumo - O objetivo deste trabalho foi comparar o desempenho produtivo, a adaptabilidade e a estabilidade de híbridos simples, triplos e duplos de milho originados de um mesmo conjunto de linhagens. Foram avaliados 10 híbridos simples, 30 híbridos triplos e 15 híbridos duplos, além de nove híbridos comerciais utilizados como testemunhas. Utilizou-se o delineamento experimental látice triplo 8x8. Os experimentos foram avaliados em 12 ambientes. O caráter avaliado foi a produtividade de espigas corrigida para 13\% de umidade. Realizaram-se as análises de variância individuais e conjunta e verificada a presença da interação genótipos x ambientes, procedeu-se à análise de estabilidade por meio da estimativa da ecovalência e do método AMMI (Additive Main Effects and Multiplicative Interaction). A produtividade média de híbridos simples, triplos e duplos, provenientes de um mesmo conjunto de linhagens, não diferiu estatisticamente, e a diferença de produtividade entre os melhores híbridos simples, duplos e triplos foi baixa (cerca de 10\%), o que torna a análise de custo e benefício necessária para a escolha de cultivares para plantio comercial. Os híbridos duplos foram os que menos contribuíram para a interação genótipos x ambientes, seguidos pelos híbridos triplos e os simples. A metodologia utilizada permite identificar híbridos estáveis e produtivos em todos os tipos de milho avaliados.

Termos para indexação: Zea mays, adaptabilidade, AMMI, estabilidade, ecovalência.

\section{Genotype by environment interaction in different types of maize hybrids}

\begin{abstract}
The objective of this work was to compare the productive performance, the adaptability and stability of single-crosses, three-way crosses and double-crosses maize hybrids originated from the same set of inbred lines. Ten single-crosses, 30 three-way crosses, and 15 double-crosses, besides nine commercial hybrids used as checks were evaluated. A $8 \times 8$ triple lattice experimental design was used in 12 environments. Ear yield corrected to $13 \%$ moisture content was the evaluated character. Individual and joint analysis of variance were performed and, in case of the presence of genotype $\mathrm{x}$ environment interaction the analysis of stability was proceeded by estimating the ecovalence and by applying the AMMI (Additive Main Effects and Multiplicative Interaction) method. The average productivity of single-crosses, three-way-crosses, and double-crosses from the same inbred lines did not statistically differ, and yield differences between the best-performance single-cross, three-way-cross and doble-cross hybrids was low (about 10\%), which indicates the cost/benefit analysis as necessary to choose cultivars for commercial cropping. Double-crosses contributed the least to the genotype $\mathrm{x}$ environment interaction, followed by three-way crosses and single-crosses. The methodology allows of identifying high yielding and stable hybrids in all the evaluated maize types.
\end{abstract}

Index terms: Zea mays, adaptability, AMMI, stability, ecovalence.

\section{Introdução}

A descoberta da heterose e a utilização de híbridos foram as principais causas do aumento em produtividade da cultura do milho. Troyer (1999) relatou que o ganho em produtividade nos Estados Unidos foi de $63 \mathrm{~kg} \mathrm{ha}^{-1}$ por ano, de 1930 a 1960, e de 110,4 $\mathrm{kg} \mathrm{ha}^{-1}$ por ano de 1960 a 1999.

A exploração do vigor híbrido, foi inicialmente feita apenas com o emprego dos híbridos duplos.
No Brasil, tem-se observado que esses híbridos vêm sendo gradativamente substituídos por híbridos triplos e simples (Rosinha, 2000). O aumento da comercialização de híbridos simples, em detrimento dos duplos e triplos, coloca o pequeno e médio produtor brasileiro em situação não muito favorável, uma vez que o gasto com a semente desses híbridos chega a ultrapassar $15 \%$ do custo total de produção da lavoura de milho.

Pesq. agropec. bras., Brasília, v.45, n.12, p.1433-1440, dez. 2010 
Apesar das mudanças que vêm ocorrendo na comercialização dos diferentes tipos de híbridos, a diferença de produtividade de grãos entre eles não é muito acentuada (Saleh et al., 2002). Assim, a adoção generalizada de um determinado tipo de híbrido deve ser feita com cautela. Nem sempre os melhores híbridos são os mais estáveis, pois diferem quanto ao grau de heterosigosidade e heterogeneidade, o que repercute diretamente na contribuição da interação genótipos $\mathrm{x}$ ambientes (Becker \& Léon, 1988).

Híbridos simples, triplos e duplos de diversas origens têm sido comparados, e os simples são os que mais contribuem para a interação de genótipos por ambientes, seguidos dos híbridos triplos e duplos (Torres, 1988; Ribeiro et al., 2000). Os trabalhos de comparação existentes, no entanto, utilizam híbridos provenientes de diferentes backgrounds genéticos, o que torna os resultados pouco informativos no que se refere à avaliação das diferenças dos diferentes tipos de híbrido, em termos de produtividade e estabilidade. Nesse caso, uma opção seria avaliar a produção e a estabilidade de diferentes tipos de híbrido obtidos de um mesmo conjunto de linhagens.

Existem diversas metodologias destinadas à avaliação da interação genótipos $\mathrm{x}$ ambientes, e a escolha do método mais adequado depende dos dados experimentais, especialmente do número de ambientes disponíveis, da precisão requerida e do tipo de informação desejada. O método da ecovalência, descrito por Wricke \& Weber (1986), estima a contribuição de cada genótipo para a interação genótipos x ambientes. Contudo, técnicas multivariadas têm ganhado espaço nesse tipo de estudo nos últimos anos, em virtude da popularização do uso de computadores e de modernos pacotes estatísticos que permitem que os complexos cálculos com álgebra de matrizes e modelos lineares, demandados por essas técnicas, sejam realizados facilmente. O método AMMI (Additive Main Effects and Multiplicative Interaction Analysis) é uma dessas técnicas multivariadas e considera modelos aditivos para os efeitos principais (genótipos e ambientes) e multiplicativos para os efeitos da interação $G \times A$ (Crossa et al., 1990).

O objetivo deste trabalho foi comparar o desempenho produtivo, a adaptabilidade e a estabilidade de híbridos simples, triplos e duplos originados de um mesmo conjunto de linhagens de milho.

\section{Material e Métodos}

Foram utilizadas cinco linhagens endogâmicas de milho, provenientes do programa de melhoramento de milho, do Departamento de Biologia, da Universidade Federal de Lavras (DBI/Ufla), com graus de endogamia de pelo menos seis gerações de autofecundação. As linhagens eram oriundas de fontes diversas e foram tomadas de forma aleatória.

No ano agrícola 2005/2006, foram gerados todos os híbridos simples possíveis. No ano agrícola de 2006/2007, com uso dos híbridos simples já sintetizados, foram obtidos todos os híbridos triplos e duplos. Para que as sementes utilizadas nos ensaios de avaliação estivessem nas mesmas condições, os híbridos simples foram novamente sintetizados. No total, foram sintetizados 10 híbridos simples, 30 híbridos triplos e 15 híbridos duplos. Todas as etapas foram realizadas na área experimental do DBI/Ufla.

Foram avaliados 64 tratamentos, correspondentes aos 55 híbridos sintetizados e nove testemunhas comerciais, das quais três eram híbridos simples (AG8060, P30F90 e DKB199), três híbridos triplos (AG5011, P30F87 e DKB350) e três híbridos duplos (AG1051, BRS2114 e DKB747).

Os 64 tratamentos foram avaliados nos municípios de Lavras, Ribeirão Vermelho e Ijaci, todos no Estado de Minas Gerais, no ano agrícola 2007/2008, em três densidades diferentes. Osexperimentos foraminstalados nas densidades populacionais de 40,60 e 80 mil plantas por hectare. No ano agrícola de 2008/2009, os experimentos foram avaliados nos mesmos três ambientes, na densidade populacional de $60 \mathrm{mil}$ plantas por hectare. Considerada a combinação local x densidade como um ambiente, os experimentos foram avaliados em 12 ambientes: nove no ano agrícola 2007/2008 e três no ano agrícola 2008/2009.

Utilizou-se o delineamento experimental látice triplo $8 \times 8$. As parcelas foram constituídas de duas linhas de $3 \mathrm{~m}$ de comprimento, espaçadas entre si de $0,50 \mathrm{~m}$.

A característica avaliada foi produtividade de espigas despalhadas, e os dados foram corrigidos para a umidade padrão de $13 \%$.

As análises de variância individual e conjunta foram realizadas com o auxílio do SAS versão 8.2, módulo SAS/STAT, procedimento GLM (SAS Institute, 2001). As médias de cada parcela foram corrigidas por covariância com o estande final. Após as análises individuais, foi realizada a análise conjunta com os 
12 ambientes de avaliação, tendo-se considerado o modelo fixo. Para essa análise, foram utilizadas as médias ajustadas de cada ambiente. No entanto, como essas médias foram obtidas com recuperação da informação do resíduo intrabloco, em cada uma delas foi necessário calcular o erro efetivo, em que as médias dos quadrados médios dos erros foram utilizadas para compor o erro efetivo médio da análise conjunta, conforme Cochram \& Cox (1957). Na análise conjunta, foram desdobradas as somas de quadrados de híbridos em híbridos experimentais, híbridos comerciais e seu contraste, e a interação deles com ambientes. Além disso, as somas de quadrados de híbridos experimentais foram desdobradas para cada um dos tipos de híbrido e para o contraste entre eles. Novamente, foram realizadas as interações desses híbridos com ambientes. Para discriminar as médias de produtividade dos diferentes tipos de híbridos, foi aplicado o teste de agrupamento de média proposto por Scott \& Knott (1974), a 5\% de probabilidade.

Uma vez constatada a presença de interação $\mathrm{G}$ x A (teste $\mathrm{F}$ significativo), procedeu-se à análise de estabilidade, que permitiu mensurar a adaptação e a estabilidade produtiva de cada híbrido. Tal avaliação foi realizada com o uso do método da ecovalência, descrito por Wricke \& Weber (1986), que estima a contribuição de cada genótipo para a interação $\mathrm{G}$ x A, e pelo método AMMI (Crossa et al., 1990), que considera os efeitos de genótipos e ambientes como aditivos e a interação G x A como multiplicativa, por meio da análise dos componentes principais (ACP). A contribuição de cada híbrido para a interação $\mathrm{G}$ x A foi obtida pela estimativa da ecovalência, expressa por,

$$
\mathrm{W}_{\mathrm{i}}^{2}=\sum\left(\mathrm{Y}_{\mathrm{ij}}-\overline{\mathrm{Y}}_{\mathrm{i} .}-\overline{\mathrm{Y}}_{. \mathrm{j}}+\overline{\mathrm{Y}}_{. .}\right)^{2}
$$

em que: $\mathrm{W}_{\mathrm{i}}{ }^{2}$ é a ecovalência associada ao híbrido $\mathrm{i} ; \mathrm{Y}_{\mathrm{ij}} \mathrm{e}$ a média fenotípica observada do híbrido i no mbiente $\mathrm{j}$; $\bar{Y}_{i}$ é a média do híbrido $\mathrm{i} ; \overline{\mathrm{Y}}_{\text {.j }}$ é a média do ambiente $\mathrm{j}$; e $\overline{\mathrm{Y}}$.. é a média geral.

Com o uso das médias ajustadas, provenientes das análises individuais, foi realizada a análise AMMI, tendo-se considerado o seguinte modelo:

$$
\mathrm{Y}_{\mathrm{ij}}=\mu+\mathrm{g}_{\mathrm{i}}+\mathrm{a}_{\mathrm{j}}+\sum_{\mathrm{k}=1}^{\mathrm{n}} \lambda_{\mathrm{k}} \gamma_{\mathrm{ik}} \alpha_{\mathrm{jk}}+\mathrm{r}_{\mathrm{ij}}+\varepsilon_{\mathrm{ij}},
$$

em que $Y_{\mathrm{ij}}$ é a produtividade média do híbrido $\mathrm{i}$, no ambiente $\mathrm{j} ; \mu$ é a produtividade média geral; $g_{\mathrm{i}}$ é o efeito do híbrido $i ; a_{j}$ é o efeito do ambiente $j ; \lambda_{\kappa}$ é o k-ésimo autovalor do eixo da ACP; $\mathrm{y}_{\mathrm{ik}}$ é o autovetor do i-ésimo híbrido, no eixo $\mathrm{k}$ da ACP; $\alpha_{\mathrm{jk}}$ é o autovetor do j-ésimo ambiente, no eixo $\mathrm{k}$ da ACP; $\mathrm{r}_{\mathrm{ij}}$ é o desvio da interação não explicada pelos componentes principais, retidos (porção ruído); $n$ é o número de eixos principais retidos para descrever o padrão da interação $\mathrm{G} \mathrm{x} \mathrm{A}$; e $\varepsilon_{\mathrm{ij}}$ é o erro experimental médio associado à observação.

As análises de ecovalência e AMMI foram realizadas por meio do programa Estabilidade, desenvolvido por Ferreira \& Zambalde (1997).

Para a escolha do modelo AMMI, foi adotado um método preditivo por meio da validação cruzada, tendo-se utilizado o procedimento "leave-one-out", proposto por Gabriel (2002). Foi aplicada a estatística Press m, para avaliar as discrepâncias entre os valores observados $\left(\mathrm{y}_{\mathrm{ij}}\right)$ e os preditos $\left(\hat{\mathrm{y}}_{\mathrm{ij}}^{\mathrm{m}}\right)$, por meio da equação:

$$
\text { Press_m }=\frac{1}{\mathrm{ge}} \sum_{\mathrm{i}=1}^{\mathrm{g}} \sum_{\mathrm{j}=1}^{\mathrm{e}}\left(\hat{\mathrm{y}}_{\mathrm{ij}}^{\mathrm{m}}-\mathrm{y}_{\mathrm{ij}}\right)^{2} \text {; }
$$

foi realizada, ainda, a correlação entre os valores observados e os valores preditos (Precorr_m), para avaliar a acurácia das predições, conforme:

$$
\text { Precorr_m }=\operatorname{Corr}\left(\mathrm{y}_{\mathrm{ij}}, \hat{\mathrm{y}}_{\mathrm{ij}}^{\mathrm{m}} \mid \forall \mathrm{i}, \mathrm{j}\right) \text {. }
$$

Após a escolha do modelo AMMI a ser utilizado, os resultados foram representados na forma gráfica de um biplot (Gabriel, 1971).

\section{Resultados e Discussão}

Nas análises de variância individuais do caráter produtividade de espiga despalhada, a relação entre o maior e o menor valor da variância do erro experimental foi de 2,74 , o que mostra que as variâncias do erro experimental podem ser consideradas homogêneas, sem impedimento para a realização da análise conjunta.

$\mathrm{Na}$ análise de variância conjunta, foram detectadas diferenças significativas, para as fontes de variação ambientes, híbridos e para a interação híbridos $\mathrm{x}$ ambientes (Tabela 1). A significância observada para os ambientes ocorreu em razão da existência de diversidade de condições edafoclimáticas, de efeitos de população e de arranjo espacial das plantas nos 12 ambientes.

A significância entre os híbridos era esperada, uma vez que eles foram sintetizados a partir de cinco 
linhagens de origens distintas, e que as testemunhas foram compostas por híbridos comerciais de diferentes empresas, ou seja, com diferentes "backgrounds" genéticos. Após o desdobramento dessa fonte de variação, verificou-se significância quanto às fontes de variação: híbridos experimentais, híbridos simples, híbridos triplos e híbridos duplos. Isso indica que os híbridos possuem índices de produtividade diferentes entre si, ou seja, em média, os híbridos têm produtividade diferenciada dentro de cada classe.

Os híbridos simples (HS), triplos (HT) e duplos (HD) mais bem classificados tiveram produtividade média de $9.274 \mathrm{~kg} \mathrm{ha}^{-1}$ (HS 7), $8.217 \mathrm{~kg} \mathrm{ha}^{-1}$ (HT 6) e $8.400 \mathrm{~kg} \mathrm{ha}^{-1}$ (HD 3), enquanto os híbridos com pior classificação tiveram produtividade de $6.857 \mathrm{~kg} \mathrm{ha}^{-1}$ (HS 5), $6.400 \mathrm{~kg} \mathrm{ha}^{-1}$ (HT 30) e $7.184 \mathrm{~kg} \mathrm{ha}^{-1}$ (HD 12), respectivamente (Tabela 2). Isto representa uma variação de produtividade de 26, 22,1 e $14,48 \%$ entre os híbridos simples, triplos e duplos com maior e menor produtividade.

A substituição dos híbridos duplos por triplos e simples tem sido cada vez mais comum, e, atualmente, os híbridos simples e triplos representam 79\% das opções de sementes disponíveis aos produtores (Cruz \& Pereira Filho, 2010). Assim, é importante levar em consideração, no momento da recomendação da

Tabela 1. Resumo da análise de variância conjunta, quanto ao caráter produtividade de espiga despalhada $\left(\mathrm{kg} \mathrm{ha}^{-1}\right) \mathrm{de}$ 64 híbridos de milho, em 12 ambientes.

\begin{tabular}{lcc}
\hline Fonte de variação & GL & Quadrado médio \\
\hline Ambientes (A) & 11 & $295.153 .800,87^{* *}$ \\
Híbridos (H) & 63 & $15.007 .721,60^{* *}$ \\
Híbridos experimentais (HE) & 54 & $8.287 .024,10^{* *}$ \\
Híbridos simples (HS) & 9 & $17.864 .148,64^{* *}$ \\
Híbridos duplos (HD) & 14 & $5.885 .104,77^{* *}$ \\
híbridos triplos (HT) & 29 & $7.015 .535,25^{* *}$ \\
Entre HE & 2 & $439.987,39$ \\
Híbridos comerciais (HC) & 8 & $25.992 .544,64^{* *}$ \\
HE vs HC & 1 & $290.046 .802,18^{* *}$ \\
HxA & 693 & $3.109 .655,10^{* *}$ \\
Híbridos experimentais x A & 594 & $2.586 .652,72^{* *}$ \\
HS x A & 99 & $2.965 .683,43 * *$ \\
HD x A & 154 & $2.039 .157,75$ \\
HT x A & 319 & $2.636 .506,79^{* *}$ \\
Entre HE x A & 22 & $3.990 .595,27^{* *}$ \\
HC x A & 88 & $4.716 .656,89^{* *}$ \\
(HE x HC) x A & 11 & $18.495 .769,56^{* *}$ \\
Erro médio & 1190 & $1.992 .864,97$ \\
\hline CV (\%) & & 18,13 \\
\hline
\end{tabular}

**Significativo a $1 \%$ de probabilidade, pelo teste $\mathrm{F}$. cultivar, se o maior potencial produtivo dos híbridos simples compensaria o maior custo de suas sementes. No presente trabalho, verificou-se que o híbrido simples mais produtivo teve superioridade de $11,4 \%$ em relação ao híbrido triplo mais produtivo e de $9,4 \%$ em relação ao híbrido duplo mais produtivo (Tabela 2), resultado este superior ao reportado por Machado et al. (2008) que observaram que o melhor híbrido simples foi 3,3\% superior ao melhor híbrido duplo.

Wricke \& Weber (1986) consideraram que, na ausência de epistasia e em uma população grande, a produtividade do melhor híbrido triplo e do melhor híbrido duplo pode ser predita por meio da produtividade dos híbridos simples. Esses autores consideram que, se os valores genotípicos dos híbridos simples seguem uma distribuição normal, a média dos diferentes tipos de híbridos possíveis de se obter, a partir deles, pode ser predita. No exemplo apresentado pelos autores, o melhor híbrido simples é $1,5 \%$ mais produtivo que o melhor híbrido triplo e $3 \%$ mais produtivo que o melhor híbrido duplo. Estes valores contrastam com os observados no presente trabalho.

A análise de variância não detectou diferenças entre os tipos de híbrido, o que indica que, em média, tanto híbridos simples quanto híbridos triplos e duplos tem produtividade semelhante. Os híbridos simples tiveram produtividade média de $7.645 \mathrm{~kg} \mathrm{ha}^{-1}$; os triplos, $7.621 \mathrm{~kg} \mathrm{ha}^{-1}$; e os duplos, $7.671 \mathrm{~kg} \mathrm{ha}^{-1}$. Os híbridos comerciais tiveram produtividade média de 8.680 $\mathrm{kg} \mathrm{ha}^{-1}$, sendo que, os híbridos simples comerciais, triplos comerciais e duplos comerciais tiveram produtividade média de $9.060,8.894$ e $8.087 \mathrm{~kg} \mathrm{ha}^{-1}$, respectivamente.

Após verificada a significância da interação $\mathrm{G}$ x A, realizou-se seu desdobramento e verificou-se que apenas a interação híbridos duplos $\mathrm{x}$ ambientes não foi significativa. Esses resultados indicam que os híbridos simples e triplos apresentaram comportamento não coincidente nos 12 ambientes, e que os híbridos duplos apresentaram comportamento coincidente, ou seja, em média, os híbridos duplos apresentaram maior estabilidade que os simples e triplos. Esse resultado é semelhante ao obtido por machado et al. (2008), que verificaram maior estabilidade de híbridos duplos em relação a híbridos simples.

Verificou-se, pelas estimativas de ecovalência obtidas dos 55 híbridos experimentais (Tabela 2), que os híbridos simples contribuíram, em média, com 
Tabela 2. Média de produtividade de espiga despalhada $\left(\mathrm{kg} \mathrm{ha}^{-1}\right)$, escore do primeiro componente principal (CP1) e estimativa de ecovalência (Wi\%) de 64 híbridos de milho em 12 ambientes ${ }^{(1)}$.

\begin{tabular}{|c|c|c|c|}
\hline Híbrido & Produtividade & CP1 & $\mathrm{Wi} \%$ \\
\hline AG8060 & $9.734 \mathrm{a}$ & $-26,91$ & - \\
\hline P30F87 & $9.687 \mathrm{a}$ & $-25,60$ & - \\
\hline HS7 & $9.274 b$ & $-9,67$ & 1,90 \\
\hline DKB747 & $9.081 \mathrm{~b}$ & $-10,46$ & - \\
\hline DKB199 & $8.980 \mathrm{~b}$ & $-29,31$ & - \\
\hline DKB350 & $8.761 \mathrm{~b}$ & $-8,40$ & - \\
\hline P30F90 & $8.468 \mathrm{c}$ & $-26,86$ & - \\
\hline HD3 & $8.400 \mathrm{c}$ & 19,70 & 2,34 \\
\hline HD2 & $8.318 \mathrm{c}$ & $-8,27$ & 0,89 \\
\hline AG1051 & $8.303 c$ & $-27,00$ & - \\
\hline AG5011 & $8.235 \mathrm{c}$ & $-29,93$ & - \\
\hline HT6 & $8.217 \mathrm{c}$ & $-7,62$ & 0,90 \\
\hline HT28 & $8.198 \mathrm{c}$ & $-2,27$ & 0,88 \\
\hline HT25 & $8.151 \mathrm{c}$ & $-3,27$ & 3,27 \\
\hline HD13 & $8.148 \mathrm{c}$ & 0,33 & 1,54 \\
\hline HT3 & $8.097 \mathrm{c}$ & 4,71 & 1,72 \\
\hline HT11 & $8.056 \mathrm{c}$ & $-17,81$ & 3,75 \\
\hline HD5 & $8.053 \mathrm{c}$ & $-2,66$ & 1,44 \\
\hline HS6 & $8.052 \mathrm{c}$ & 40,58 & 6,23 \\
\hline HT29 & $8.022 \mathrm{c}$ & 3,79 & 3,07 \\
\hline HT2 & $7.984 \mathrm{c}$ & $-14,77$ & 1,48 \\
\hline HT4 & $7.961 \mathrm{c}$ & $-0,68$ & 1,39 \\
\hline HD7 & $7.946 \mathrm{c}$ & 8,77 & 1,29 \\
\hline HT9 & $7.940 \mathrm{c}$ & 4,61 & 2,52 \\
\hline HT13 & $7.935 \mathrm{c}$ & $-9,05$ & 1,44 \\
\hline HT26 & $7.911 \mathrm{c}$ & 5,72 & 1,52 \\
\hline HS3 & $7.909 \mathrm{c}$ & $-5,97$ & 1,64 \\
\hline HS1 & $7.867 \mathrm{c}$ & 6,56 & 0,87 \\
\hline HT1 & $7.817 \mathrm{~d}$ & $-13,26$ & 1,64 \\
\hline HT19 & $7.816 \mathrm{~d}$ & 10,24 & 1,21 \\
\hline HD6 & $7.772 \mathrm{~d}$ & 12,65 & 2,10 \\
\hline HS4 & $7.731 d$ & $-8,29$ & 1,84 \\
\hline HT15 & $7.717 \mathrm{~d}$ & $-4,88$ & 1,62 \\
\hline HT27 & $7.717 \mathrm{~d}$ & 18,17 & 2,58 \\
\hline HT12 & $7.687 \mathrm{~d}$ & 12,79 & 1,15 \\
\hline HT7 & $7.641 \mathrm{~d}$ & $-28,28$ & 3,41 \\
\hline HD4 & $7.616 \mathrm{~d}$ & 0,58 & 1,34 \\
\hline HT5 & $7.610 \mathrm{~d}$ & 3,96 & 1,35 \\
\hline HD10 & $7.585 \mathrm{~d}$ & 14,44 & 1,15 \\
\hline HT20 & $7.566 \mathrm{~d}$ & 7,65 & 1,53 \\
\hline HT18 & $7.566 \mathrm{~d}$ & 13,60 & 1,03 \\
\hline HS2 & $7.555 \mathrm{~d}$ & $-0,67$ & 1,08 \\
\hline HT22 & $7.538 \mathrm{~d}$ & $-5,93$ & 3,60 \\
\hline HD1 & $7.512 \mathrm{~d}$ & $-1,21$ & 1,87 \\
\hline HD14 & $7.434 d$ & 13,95 & 1,57 \\
\hline HT8 & $7.427 \mathrm{~d}$ & $-5,44$ & 1,77 \\
\hline HD15 & $7.379 \mathrm{~d}$ & 19,05 & 2,42 \\
\hline HT17 & $7.359 \mathrm{~d}$ & $-14,20$ & 2,28 \\
\hline HS8 & $7.303 \mathrm{e}$ & 6,04 & 2,11 \\
\hline HD9 & $7.262 \mathrm{e}$ & 10,01 & 0,94 \\
\hline HT21 & $7.246 \mathrm{e}$ & 27,12 & 2,55 \\
\hline HD11 & $7.236 \mathrm{e}$ & 4,90 & 0,87 \\
\hline HT10 & $7.236 \mathrm{e}$ & $-6,12$ & 1,24 \\
\hline HD8 & $7.219 \mathrm{e}$ & 5,63 & 1,54 \\
\hline HD12 & $7.184 \mathrm{e}$ & 17,92 & 1,63 \\
\hline HT16 & $7.058 \mathrm{e}$ & 13,52 & 1,06 \\
\hline HT23 & $7.033 \mathrm{e}$ & 8,04 & 2,19 \\
\hline HS9 & $6.971 \mathrm{e}$ & 2,36 & 1,31 \\
\hline HS10 & $6.935 \mathrm{e}$ & 18,99 & 1,47 \\
\hline НT24 & $6.918 \mathrm{e}$ & 3,33 & 2,11 \\
\hline BRS2114 & $6.877 \mathrm{e}$ & $-3,14$ & - \\
\hline HS5 & $6.857 \mathrm{e}$ & $-1,86$ & 2,25 \\
\hline HT14 & $6.812 \mathrm{e}$ & 6,47 & 0,87 \\
\hline HT30 & $6.400 \mathrm{e}$ & 13,59 & 1,21 \\
\hline
\end{tabular}

${ }^{(1)}$ Médias seguidas de letras iguais não diferem entre si, pelo teste de Scott-Knott a 5\% de probabilidade.
$2,07 \%$ para a interação $\mathrm{G} \times \mathrm{A}$, os triplos com 1,84\%, e os duplos com 1,52\%. Essa diferença pode aparentar ser pequena, porém, corresponde a estimativas de $\mathrm{W}_{\mathrm{i}}$ $26,15 \%$ inferiores para os híbridos duplos e $10,77 \%$ inferiores para os híbridos triplos, em relação às obtidas para os híbridos simples. Adiferença entre as estimativas $\mathrm{W}_{\mathrm{i}}$ de híbridos triplos e duplos foi de 17,23\%. Quando observadas as menores contribuições individuais, de cada tipo de híbrido, para a interação $\mathrm{G}$ x A, nota-se que os híbridos que menos contribuíram foram o HS1, o HT14 e o HD11, todos com contribuição de $0,87 \%$. Por meio da análise AMMI, espera-se capturar grande parte do padrão estrutural da $\mathrm{SQ}_{\mathrm{GxA}}$ nos primeiros componentes. Em concordância com esse modelo, a interação $\mathrm{G}$ x A original pode ser decomposta em 11 componentes (posto da matriz $\mathrm{G} \times \mathrm{A}$ ), dos quais os três primeiros foram significativos $(\mathrm{p}<0,01)$. Segundo a regra de Gollob, isto levaria à escolha do modelo AMMI3, e seriam necessários três eixos principais para explicar a interação de forma significativa. Nesse caso, seria possível a construção de três gráficos, com os três componentes principais combinados dois a dois.

Dias \& Krzanowski (2003) compararam algumas metodologias de seleção do modelo AMMI e argumentam que testes baseados em distribuições F geralmente têm suposições questionáveis, e que modelos de validação cruzada como o "leave-one-out" fazem o uso mais eficiente dos dados e provêm modelos mais parcimoniosos que, em geral, devem ser recomendados.

O procedimento padrão para a validação cruzada é subdividir a matriz de interação em vários grupos, apagar um grupo por vez, avaliar os parâmetros de predição por meio dos dados restantes e predizer os dados apagados. Gabriel (2002) sugere que o número ótimo de componentes principais é aquele em que as discrepâncias entre os valores observados e preditos (Press_m) são os menores e com a maior correlação entre os valores observados e preditos (Precorr_m). Assim, o modelo selecionado foi o AMMI1 (Tabela 3). Este modelo possui 147 graus de liberdade e é uma vez e meia mais parcimonioso que o AMMI2, pois contém uma vez e meia mais graus de liberdade de que o AMMI1. O primeiro componente da interação explorou $23,2 \%$ da interação híbridos $\mathrm{x}$ ambientes em apenas $10,53 \%$ dos graus de liberdade da interação. Segundo Dias \& Krzanowski (2003), os maiores componentes da interação são considerados, por 
avaliação preditiva, apenas ruídos para a proposição da predição de produtividade e deveriam ser combinados com o resíduo.

A interpretação de um biplot AMMI1 quanto à interação $\mathrm{G} \times \mathrm{A}$ é feita observando-se a magnitude e o sinal dos escores de genótipos e ambientes para o eixo da interação. Logo, os pontos situados na faixa horizontal, em torno de zero, em relação ao CP1,

Tabela 3. Resumo da análise AMMI, soma de quadrados (SQ) da interação genótipo $\mathrm{x}$ ambiente $(\mathrm{G} \times \mathrm{A})$ e a percentagem explicada [SQ (GxA)], e discrepância (Press_m) e correlação (Precorr_m) entre valores observados e preditos.

\begin{tabular}{lccccc}
\hline FV & GL & SQ & SQ (G x A) & Press_m & Precorr_m \\
\hline G x A & 693 & 2154950906 & 100 & - & - \\
CP 1 & 73 & 500141441,9 & 23,21 & 0,9768 & 0,8122 \\
CP 2 & 71 & 315143909,2 & 37,83 & 0,9916 & 0,8090 \\
CP 3 & 69 & 240112672 & 50,64 & 0,9925 & 0,8083 \\
Resíduo & 1190 & 2371509313 & - & - & - \\
\hline
\end{tabular}

correspondem aos genótipos mais estáveis (Carbonell et al., 2004; Maia et al., 2006). Entre estes estão os híbridos HS2, HS5, HS9, HT3, HT4, HT5, HT9, HT25, HT28, HT29, HD1, HD5, HD11 e HD13. Esses híbridos mostraram-se estáveis nos ambientes testados; entretanto, para fins de recomendação de cultivares, genótipos estáveis também devem apresentar alta produtividade (Figura 1).

Alvarez \& Eyhérabide (1996) subdividem o biplot AMMI1 em quatro classificações quanto a genótipos e ambientes como: de produtividade média elevada e de baixa interação $\mathrm{G} \times \mathrm{A}$; de baixa produtividade e estável; de produtividade elevada e maior interação $\mathrm{G} \times \mathrm{A}$; de baixa produtividade e instável. Assim, destacam-se como estáveis e com produtividade média acima da média geral os híbridos HT28, HT25, HD13, HT3, HD5, HT29, HT4 e HT9, em ordem decrescente de produtividade.

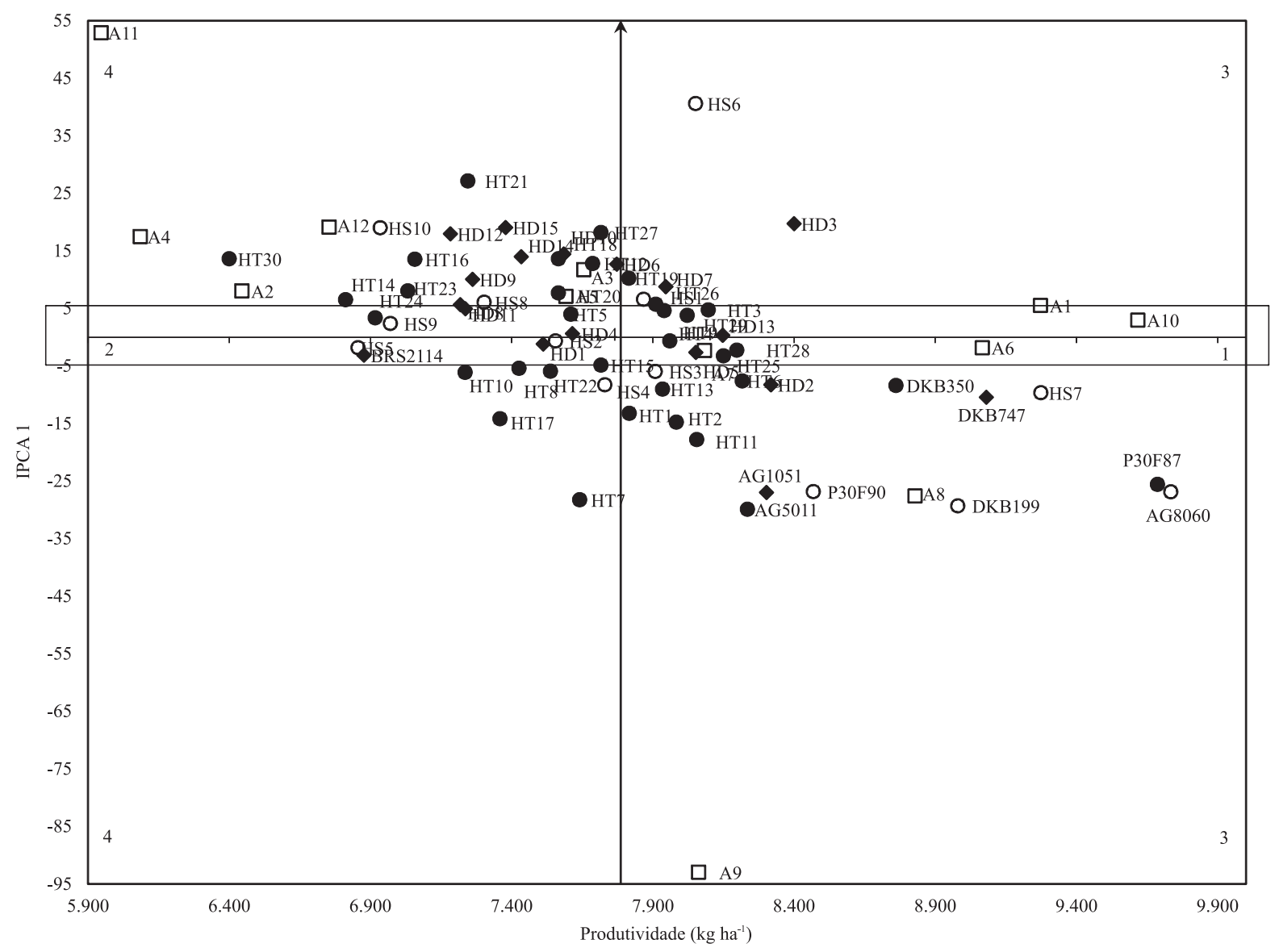

Figura 1. Biplot da análise AMMI1 quanto à produtividade de espigas despalhadas $\left(\mathrm{kg} \mathrm{ha}^{-1}\right)$, em 64 híbridos de milho e 12 ambientes: (o), híbridos simples; (•), triplos; (४), duplos; e (ם), ambientes. Os números de 1 a 4 representam zonas de classificação de genótipos e ambientes. 
O híbrido HS7 foi o mais produtivo $\left(9.274 \mathrm{~kg} \mathrm{ha}^{-1}\right)$, porém com estabilidade intermediária e interação específica e positiva com os ambientes A6 e A8.

Deve-se ressaltar que o híbrido simples mais produtivo (HS7) foi superior à média dos híbridos simples utilizados como testemunhas, e os híbridos duplos HD3, HD2 e HD13 tiveram desempenho superior à média dos híbridos duplos utilizados como testemunha. Entre esses híbridos duplos, o HD13 mostrou-se, ainda, estável nos ambientes de avaliação. Portanto, esses híbridos devem ser considerados promissores.

A escolha do tipo de híbrido a ser utilizado depende de uma série de fatores como as condições socioeconômicas de cada produtor e região. Souza Junior (2001) argumenta que, para um caráter complexo como a produtividade de grãos, a variância fenotípica é muito afetada pelas variações ambientais e pelas interações G x A e que, dessa forma, a variância fenotípica dos diferentes tipos de híbridos apresentarão valores muito próximos. Assim, a resposta à seleção de diferentes tipos de híbridos será função de suas variâncias genéticas. Assim, para híbridos de uma mesma fonte, os simples serão superiores aos triplos, e estes, por sua vez, superiores aos duplos.

No presente trabalho, verificou-se que híbridos estáveis e produtivos podem ser selecionados, independentemente da estrutura genética. O híbrido simples HS7 apresentou estabilidade média e produtividade superior. Entre os híbridos triplos e híbridos duplos, não houve diferença de produtividade entre os dois mais produtivos, conforme teste de Scott \& Knott (1974), a 5\% de probabilidade. Assim, para agricultores que utilizam altos níveis de tecnologia, com vistas à máxima produtividade e uniformidade, a utilização de híbridos simples é justificável. Contudo, a utilização dos híbridos duplos pode ser vantajosa para condições de menor investimento (Ribeiro et al., 2000).

\section{Conclusões}

1. A produtividade média de híbridos simples, triplos e duplos provenientes de um mesmo conjunto de linhagens não apresenta diferença significativa.

2. A diferença de produtividade entre os melhores híbridos simples, duplos e triplos é baixa, em torno de $10 \%$, o que torna a análise de custo e benefício necessária para a escolha de cultivares para plantio comercial.

3. Em média, os híbridos duplos são os que menos contribuem para a interação genótipos x ambientes, seguidos pelos híbridos triplos e simples.

4. A metodologia utilizada permite identificar híbridos simples, triplos e duplos produtivos e estáveis.

\section{Agradecimentos}

Ao Conselho Nacional de Desenvolvimento Científico e Tecnológico (CNPq), pela concessão de bolsa.

\section{Referências}

ALVAREZ, M. del P.; EYHÉRABIDE, G.H. Estabilidad del rendimiento de cultivares de híbridos comerciales de maíz en el área de la EEA Pergamino. Revista de Tecnología Agropecuaria, v.1, p.17-21, 1996.

BECKER, H.C.; LÉON, J. Stability analysis in plant breeding. Plant Breeding, v.101, p.1-23, 1988.

CARBONELL, S.A.M.; AZEVEDO FILHO, J.A. de; DIAS, L.A. dos S.; GARCIA, A.A.F.; MORAIS, L.K. de. Common bean cultivars and lines interactions with environments. Scientia Agricola, v.61, p.169-177, 2004.

COCHRAM, W.G.; COX, S.M. Experimental designs. $2^{\text {nd }}$ ed. New York: Wiley, 1957. 611p.

CROSSA, J.; GAUCH, H.G.; ZOBEL, R.W. Additive main effects and multiplicative interaction analysis of two international maize cultivar trials. Crop Science, v.30, p.493-500, 1990.

CRUZ, J.C.; PEREIRA FILHO, I.A. Milho - cultivares para 2009/2010. Disponível em: $<$ http://www.cnpms.embrapa.br/milho/ cultivares/index.php $>$. Acesso em: 14 jun. 2010.

DIAS, C.T.S.; KRZANOWSKI, W.J. Model selection and cross validation in additive main effect and multiplicative interaction models. Crop Science, v.43, p.865-873, 2003.

FERREIRA, D.F.; ZAMBALDE, A.L. Simplificação das análises de algumas técnicas especiais da experimentação agropecuária no mapgem e softwares correlatos. In: CONGRESSO DA SOCIEDADE BRASILEIRA DE INFORMÁTICA APLICADA A AGROPECUÁIRA E AGROINDÚSTRIA, 1., 1997, Belo Horizonte. Anais. Belo Horizonte: Agrosoft, 1997. v.1, p.54-60.

GABRIEL, K.R. Le biplot: outil d'exploration des données multidimensionnelles. Journal de la Société Française de Statistique, v.143, p.5-55, 2002.

GABRIEL, K.R. The biplot graphic display of matrices with application to principal components analysis. Biometrika, v.58, p.453-467, 1971.

MACHADO, J.C.; SOUZA, J.C. de; RAMALHO, M.A.P.; LIMA, J.L. Estabilidade de produção de híbridos simples e duplos 
de milho oriundos de um mesmo conjunto gênico. Bragantia, v.67, p.627-631, 2008.

MAIA, M.C.M.; VELLO, N.A.; ROCHA, M. de M.; PINHEIRO, J.B.; SILVA JÚNIOR, N.F. da. Adaptabilidade e estabilidade de linhagens experimentais de soja selecionadas para caracteres agronômicos através de método uni-multivariado. Bragantia, v.65, p.215-226, 2006.

RIBEIRO, P.H.E.; RAMALHO, M.A.P.; FERREIRA, D.F. Adaptabilidade e estabilidade de genótipos de milho em diferentes condições ambientais. Pesquisa Agropecuária Brasileira, v.35, p.2213-2222, 2000.

ROSINHA, R.O. Estratégias competitivas e reestruturação da indústria de sementes no Brasil: a análise do segmento do milho. 2000. 143p. Dissertação (Mestrado) - Universidade Federal de Minas Gerais, Belo Horizonte.

SALEH, G.B.; ABDULlAH, D.; ANUAR, A.R. Performance, heterosis and heritability in selected tropical maize single, double and three-way cross hybrids. Journal of Agricultural Science, v.138, p.21-28, 2002.
SAS INSTITUTE. SAS user's guide: statistics. Versão 8.2. Cary: SAS Institute, 2001. 1028p.

SCOTT, A.J.; KNOTT, M.A. A cluster analysis method for grouping means in the analysis of variance. Biometrics, v.30, p.507-512, 1974.

SOUZA JUNIOR, C.L. de. Melhoramento de espécies alógamas. In: NASS, L.L.; VALOIS, A.C.C.; MELO, I.S. de; VALADARES-INGLIS, M.C. Recursos genéticos e melhoramento. Rondonópolis: Fundação mT, 2001. p.159-199.

TORRES, R.A. de A. Estudo da estabilidade fenotípica de cultivares de milho (Zea mays L.). 1988. 133p. Tese (Doutorado) - Escola Superior de Agricultura Luiz de Queiroz, Piracicaba.

TROYER, A.F. Background of U.S. hybrid corn. Crop Science, v.39, p.601-626, 1999.

WRICKE, G.; WEBER, W.E. Quantitative genetics and selection in plant breeding. New York: Walter de Gruyter, 1986. $406 \mathrm{p}$.

Recebido em 25 de agosto de 2010 e aprovado em 10 de novembro de 2010 